\title{
Measurement of biliary alkaline phosphatase by mini-column chromatography and by electrophoresis and its application to the detection of liver metastases in patients with breast cancer
}

\author{
CAROL KARMEN, PD MAYNE, A YING FOO, S PARBHOO, SB ROSALKI
}

From the Department of Chemical Pathology and the Academic Department of Surgery, Royal Free Hospital and School of Medicine, London NW3

SUMMARY We describe a simple, rapid, and reproducible ion exchange mini-column chromatographic method for the quantitative measurement of biliary alkaline phosphatase in plasma. We have used this method to evaluate a cellulose acetate electrophoretic method, which was used to assess the value of measuring biliary alkaline phosphatase in 85 patients with breast cancer investigated for possible hepatic metastases. Biliary alkaline phosphatase activity was abnormal in 19 of 24 patients $(79 \%)$ with liver metastases, but abnormalities were also found in 12 of 61 patients $(20 \%)$ without hepatic metastases; in only $37 \%$ of patients with positive test results was this a consequence of liver metastases. For the identification of liver metastases, therefore, the method has useful sensitivity but limited specificity. Neither sensitivity nor specificity was significantly better than that of plasma $\gamma$-glutamyltransferase activity, which was measured concomitantly.

The biliary isoenzyme of alkaline phosphatase (EC 1.1.1.3) is a high molecular mass fraction (greater than $2 \mathrm{MDa}$ ) derived from plasma membrane fragments of cells lining the biliary tract or from the aggregation in bile or plasma of liver alkaline phosphatase with lipid and protein. ${ }^{12}$ Biliary alkaline phosphatase is absent from plasma in healthy subjects and its measurement in plasma has been suggested as a sensitive test for cholestatic liver disease ${ }^{2}$ and for the detection of hepatic metastases. ${ }^{34}$ The method used most often is electrophoresis, usually on cellulose acetate membrane, followed by incubation with a chromogenic alkaline phosphatase substrate and densitometric scanning of the coloured product; biliary alkaline phosphatase is located in the $\alpha_{1}$-globulin position. ${ }^{5}$ This method has been criticised, however, for its imprecision, ${ }^{6}$ and its accuracy has hitherto been uncertain.

Ion exchange chromatography has been shown previously to separate biliary from other alkaline phosphatase isoenzymes. By modification of one of these techniques ${ }^{6}$ we have developed a mini-column chromatographic method for the separation and

Accepted for publication 26 October 1983. quantification of biliary alkaline phosphatase which is suitable for use in the diagnostic laboratory. With this procedure we have evaluated the performance of a cellulose acetate electrophoretic method. ${ }^{7}$ The latter was also used in a clinical study designed to assess the claim that biliary alkaline phosphatase is more sensitive than the plasma enzyme $\gamma$-glutamyltransferase (EC 2.3.2.2.) for the detection of hepatic metastases. ${ }^{8}$

\section{Material and methods}

DETERMINATION OF TOTAL ALKALINE

PHOSPHATASE ACTIVITY

Total alkaline phosphatase activity was determined using 4-nitrophenylphosphate as substrate and diethanolamine as buffer. $\gamma$-glutamyltransferase activity was determined using $\gamma$ glutamyl-4-nitroanilide as substrate and Tris (hydroxymethyl)-aminomethane-glycylglycine buffer. Both enzymes were measured at $30^{\circ} \mathrm{C}$ by the proposed methods of the Association of Clinical Biochemists 9 adapted for the Abbott ABA $100 \mathrm{Bi}$ chromatic Analyser (Abbott Laboratories, Dallas, 
Texas, USA). The adult upper reference limits in this laboratory for these enzymes are $205 \mathrm{U} / \mathrm{l}$ for both men and women for alkaline phosphatase and $45 \mathrm{U} / \mathrm{l}$ for men and $25 \mathrm{U} / \mathrm{l}$ for women for $\gamma$-glutamyltransferase.

\section{CHROMATOGRAPHIC METHOD FOR BILIARY}

ALKALINE PHOSPHATASE

Ten grams of the anion exchange resin diethylaminoethyl cellulose (DEAE cellulose medium mesh, Sigma Chemical Co, Poole, UK) was washed twice with $200 \mathrm{ml}$ of Tris (hydroxymethyl)aminomethane buffer, $10 \mathrm{mmol} / \mathrm{l}, \mathrm{pH} 7 \cdot 5$, containing sodium chloride $(\mathrm{NaCl}), 100 \mathrm{mmol} / \mathrm{l}$ (= buffer A). The resin was allowed to settle and excess buffer removed by aspiration to give a thick slurry which was deaerated by means of a water pump. The resin was poured into small chromatography "minicolumns" of $80 \mathrm{~mm}$ height and $8 \mathrm{~mm}$ internal diameter (Worthington Diagnostics, Freehold, New Jersey, USA) and allowed to settle to a packed height of $60 \mathrm{~mm}$. The amount of resin chosen was sufficient to fill 20 mini-columns. The resin was again washed with two $6 \mathrm{ml}$ additions of buffer $\mathrm{A}$, which were allowed to drain to the resin surfaces.

Plasma $(200 \mu \mathrm{l})$ was loaded onto the column and allowed to soak into the resin; the displaced buffer was discarded. Buffer A $(6 \mathrm{ml})$ was added to the top of the resin, and the eluate collected as a single $6 \mathrm{ml}$ fraction (fraction I). Six millilitres of a second buffer of higher $\mathrm{NaCl}$ concentration (Tris $10 \mathrm{mmol} / \mathrm{l} ; \mathrm{NaCl}$ $300 \mathrm{mmol} / \mathrm{l}, \mathrm{pH} 7 \cdot 5=$ buffer $\mathrm{B}$ ) was then added and a second $6 \mathrm{ml}$ eluate volume collected (fraction II). Alkaline phosphatase activity of both fractions was measured using a 1:10 sample to substrate volume ratio in place of the $1: 100$ used for total alkaline phosphatase measurement. Biliary alkaline phosphatase activity was calculated by dividing the activity of fraction II by the sum of the activities of fractions I and II and multiplying this quotient by the total alkaline phosphatase activity of the plasma sample.

\section{ELECTROPHORETIC METHOD}

Samples $(1 \mu \mathrm{l})$ were electrophoresed on cellulose acetate membrane (Sephraphore III, Gelman Instrument Co, Ann Arbor, Mich, USA) using Tris-barbitone buffer (Gelman high resolution buffer $\mathrm{pH} 8 \cdot 8$, ionic strength $0 \cdot 05$ ) at $250 \mathrm{~V}$ for $60 \mathrm{~min}$ at $4^{\circ} \mathrm{C}$. Alkaline phosphatase activity was shown by incubating the membrane for $60 \mathrm{~min}$ at $37^{\circ} \mathrm{C}$ with a chromogenic enzyme substrate, $p$-toluidinium 5-bromo-4-chloro-3-indoxylphosphate (Sigma Chemical Co, Poole, UK), final concentration $1.25 \mathrm{mmol} / \mathrm{l}$, incorporated together with 2amino-2-methyl propanediol (AMPD) buffer, 1 $\mathrm{mol} / \mathrm{l}, \mathrm{pH} 10 \cdot 2$, and magnesium sulphate, $1 \mathrm{mmol} / \mathrm{l}$, in a $10 \mathrm{~g} / 1$ agar gel. The membrane was then fixed with $5 \% \mathrm{vol} / \mathrm{vol}$ acetic acid, washed with distilled water, and dried overnight. After inspection to ensure satisfactory separation and staining, the isoenzymes were quantified by reflectance densitometric scanning at $600 \mathrm{~nm}$ using a Corning 720 Densitometer (Corning Medical, Medifield, Mass, USA). The detection limit for alkaline phosphatase activity using this method is 5-10 U/l.

Biliary alkaline phosphatase is well separated from the other alkaline phosphatase isoenzymes by this method. Its activity was calculated by multiplying the percentage of this fraction by sample total alkaline phosphatase activity.

\section{PATIENT SAMPLES \\ Comparison studies}

One hundred and eleven consecutive plasma samples with raised total alkaline phosphatase and $\gamma$-glutamyltransferase activities as measured on the Technicon SMAC Analyser (Technicon Instruments Corporation, Tarrytown, NY, USA) were chosen for comparison studies of the electrophoretic and ion exchange chromatographic methods.

\section{Clinical studies}

Plasma samples were obtained from 85 women with previous histologically proven breast carcinoma and investigated for possible hepatic metastases. Patients were examined at intervals of about six months over an 18 month period and suspected hepatic metastases diagnosed clinically and confirmed by ultrasonic or scintigraphic examination or by computed tomography. At the time of metastases diagnosis all patients were anicteric and none had hepatomegaly.

\section{Results}

\section{DEVELOPMENT AND EVALUATION OF}

CHROMATOGRAPHIC METHOD

Elution profiles were studied by eluting $0.2 \mathrm{ml}$ plasma samples with 10 separate $1 \mathrm{ml}$ additions of buffer $\mathrm{A}$, followed by 10 separate $1 \mathrm{ml}$ additions of buffer $\mathrm{B}$, the eluates being collected as 20 consecutive individual $1 \mathrm{ml}$ fractions. Enzyme activity was present in fractions I to IV and fractions XI to XVI, but was absent from the other fractions (Fig. 1).

The enzyme-containing eluates were pooled, concentrated $(\times 50)$ using a Minicon concentrator (Amicon Corporation, Lexington, Ma, USA), and electrophoresed on cellulose acetate. Fractions I and II from several plasma samples were similarly studied. Eluates 1 to 4 (Fig. 2) and fraction I contained negligible biliary alkaline phosphatase, but hepatic, intestinal, and bone isoenzymes could be 


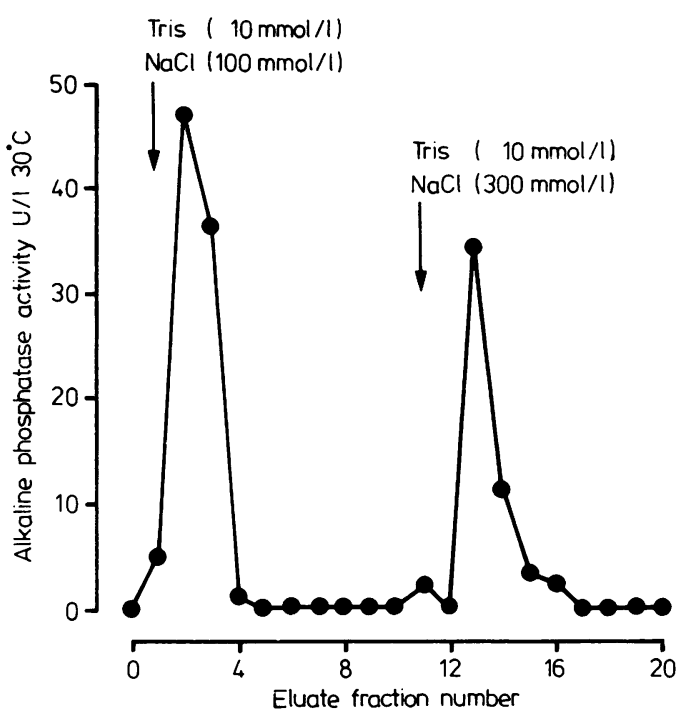

Fig. 1 Elution profile of plasma alkaline phosphatase separated by DEAE-cellulose chromatography.

detected. Eluates 11-16 and fraction II contained only biliary alkaline phosphatase. The activities of these concentrated eluates was such that less than $1 \%$ cross contamination would have been detectable by electrophoresis.

To ensure that elution was complete a third buffer of higher ionic strength containing Tris $10 \mathrm{mmol} / \mathrm{l}$ and $\mathrm{NaCl} 450 \mathrm{mmol} / \mathrm{l}, \mathrm{pH} \mathrm{7.5}$, was used. The addition of this buffer to the column in place of buffer $B$ did not result in a further increase in activity of fraction II, nor did its addition after buffer B result in elution of alkaline phosphatase in subsequent eluates.

On the basis of these elution patterns and electrophoretic observations, for the final method a single $6 \mathrm{ml}$ eluate was collected after elution with
$6 \mathrm{ml}$ buffer $\mathrm{A}$, and a further single $6 \mathrm{ml}$ eluate was collected after elution with $6 \mathrm{ml}$ buffer $B$.

There was a linear relation between sample volume and eluate activity for both fractions I and II for sample volumes up to $1 \mathrm{ml}$ of $400 \mathrm{U} / \mathrm{l}$ total activity and $60 \mathrm{U} / 1$ biliary alkaline phosphatase. With the usual $0.2 \mathrm{ml}$ sample volume, samples of alkaline phosphatase activity up to five times these values may be applied undiluted without exceeding the separation capacity of the column. Samples with greater activity should be diluted with buffer A. A sample volume of $0.2 \mathrm{ml}$ was chosen in order to conserve plasma.

The percentage alkaline phosphatase recovery was calculated by comparing the activity in fractions I and II (corrected for sample dilution) with the total alkaline phosphatase activity of the original specimen. The mean recovery on 112 plasma samples was $81 \cdot 5 \%$ (SD $11 \cdot 1 \%$ ). Recovery of alkaline phosphatase was independent of the percentage biliary alkaline phosphatase composition, which ranged up to $40 \%$ of total activity.

To see if recovery could be improved by addition of magnesium ions, magnesium sulphate was included at a final concentration of $1 \mathrm{mmol} / \mathrm{l}$ in buffers $A$ and $B$, and eluate alkaline phosphatase activity was compared with that obtained when magnesium sulphate was absent from these buffers. The effect of zinc ions on eluate alkaline phosphatase activity was determined by including zinc sulphate (1 mmol/l) and $\mathrm{N}$-hydroxyethylethylene diamine triacetic acid (HEDTA) sodium salt (Sigma Chemical Co, Poole, UK), $1 \mathrm{mmol} / \mathrm{l}$, in the diethanolamine buffer used for the determination of total alkaline phosphatase activity. The effect of the addition of HEDTA alone $(1 \mathrm{mmol} / \mathrm{l})$ to the enzyme substrate buffer was also assessed. Neither magnesium nor zinc ions enhanced the alkaline phosphatase activity of the eluate fractions or altered the percent recovery.

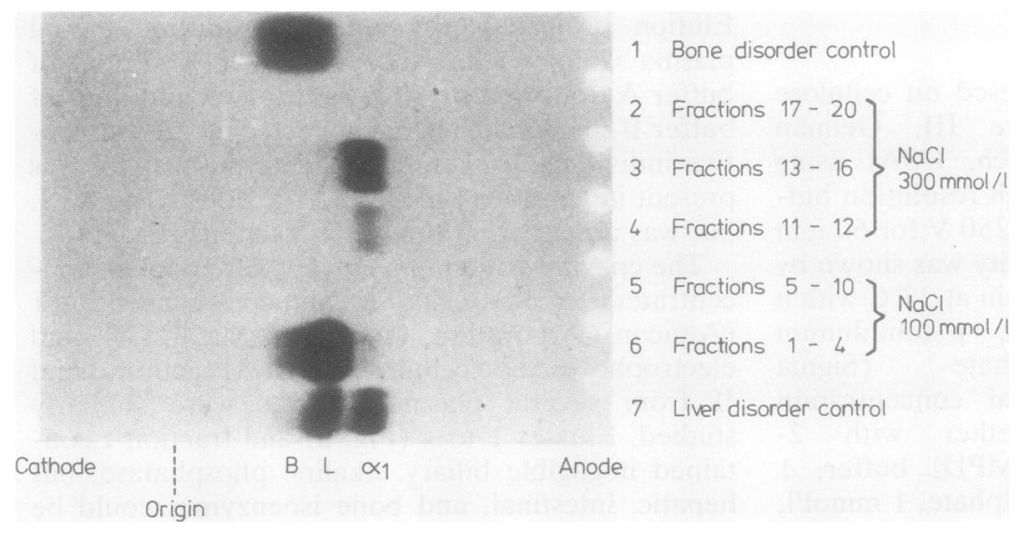

Fig. 2 Alkaline phosphatase isoenzyme composition of pooled $D E A E$-cellulose eluates concentrated $\times 50 . B=$ bone, $L=$ liver and $\alpha_{1}=$ biliary alkaline phosphatase. 
The within batch reproducibility was studied by repeated analysis of a single plasma sample on nine individual columns. A coefficient of variation of $6.4 \%$ was obtained at a mean biliary alkaline phosphatase activity of $63.1 \mathrm{U} / \mathrm{l}$. The between batch reproducibility was determined by repeat analysis of 20 samples from five batches with biliary alkaline phosphatase activity ranging from $10 \mathrm{U} / 1$ to $209 \mathrm{U} / \mathrm{l}$. A coefficient of variation of $10.4 \%$ was obtained.

\section{EVALUATION OF ELECTROPHORETIC METHOD}

The within batch reproducibility was assessed by electrophoresis of a single sample 16 times on two cellulose acetate membranes. A coefficient of variation of $3.2 \%$ was obtained at a mean biliary alkaline phosphatase activity of $167 \mathrm{U} / 1$, representing $41 \%$ of total alkaline phosphatase. Between batch reproducibility ( 10 batches) on the same sample gave a coefficient of variation of $5.8 \%$.

\section{COMPARISON OF METHODS}

There was a good correlation between the electrophoretic method $(y)$ and the chromatographic method $(x)$, with a correlation coefficient of 0.84 and a regression equation (by Deming's method) of $y=2.03 x-12.76$ (Fig. 3). The values for biliary alkaline phosphatase activity obtained by the electrophoretic method were about twice those obtained by the ion exchange procedure.

In six samples biliary alkaline phosphatase activity could not be identified by electrophoresis but biliary alkaline phosphatase activity up to $34 \mathrm{U} / \mathrm{l}$ was measurable by the chromatographic method.

\section{RESULTS OF CLINICAL STUDIES}

Patients with carcinoma of the breast

Eighty five patients were studied. Ten patients had hepatic metastases, in seven of whom biliary alkaline phosphatase was present and in nine of whom $\gamma$-glutamyltransferase activity was increased. Fourteen patients developed hepatic metastases during the 18 month follow up. Seven of these had initially shown biliary alkaline phosphatase activity and four had increased $\gamma$-glutamyltransferase activity. The median time for the development of metastases (10 months) was independent of increased biliary alkaline phosphatase or $\gamma$-glutamyltransferase activity at initial examination. Of the 24 patients with metastases at the start of the study or during follow up, 19 patients $(79 \%)$ showed biliary alkaline phosphatase and 19 patients $(79 \%)$ had raised $\gamma$-glutamyltransferase activity at the time of diagnosis of metastases.

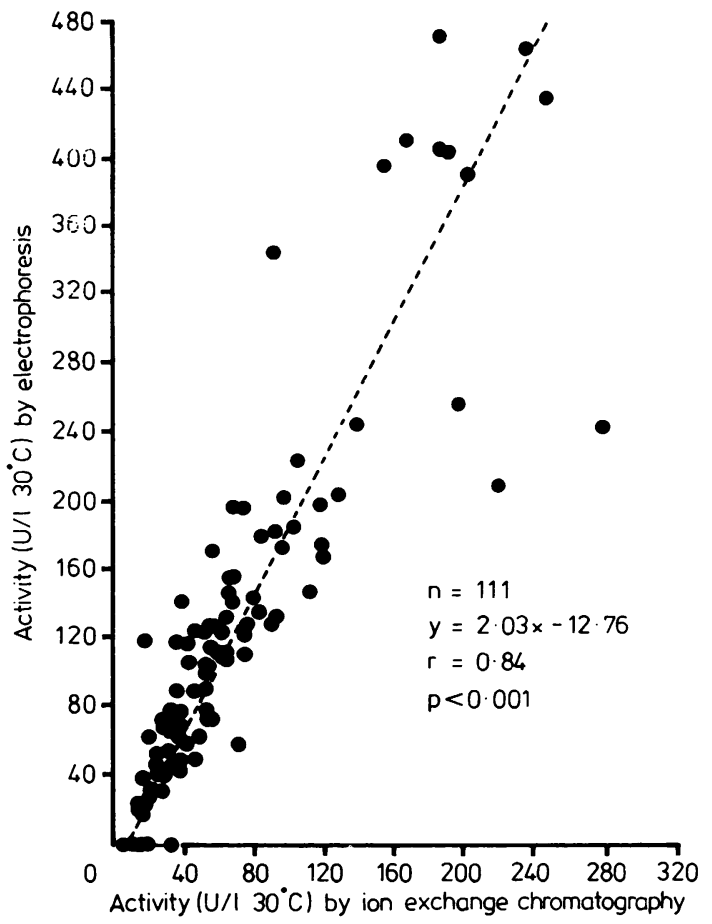

Fig. 3 Comparison of biliary alkaline phosphatase measured by electrophoresis and by ion exchange chromatography.

In 61 patients there was no evidence of hepatic metastases initially or throughout the 18 month follow up. Of these patients $12(20 \%)$ had detectable biliary alkaline phosphatase and $20(33 \%)$ had increased $\gamma$-glutamyltransferase activity. Possible causes for these false positive enzyme results included excessive alcohol consumption (two patients), gall stones (two patients), right heart failure secondary to chronic obstructive airways dis-

Diagnostic value of biliary alkaline phosphatase and $\gamma$-glutamyltransferase determination for the detection of hepatic metastases present at the time of initial examination

\begin{tabular}{lll}
\hline & $\begin{array}{l}\text { Biliary } \\
\text { alkaline } \\
\text { phosphatase }\end{array}$ & $\begin{array}{l}\text { r-glutamyl- } \\
\text { transferase }\end{array}$ \\
\hline Test sensitivity & $70 \%$ & $90 \%$ \\
$\begin{array}{l}\text { Test specificity } \\
\text { Predictive value of } \\
\text { positive test (PV+) }\end{array}$ & $\mathbf{8 0 \%}$ & $67 \%$ \\
$\begin{array}{c}\text { Predictive value of } \\
\text { negative test (PV-) }\end{array}$ & $94 \%$ & $31 \%$ \\
\hline
\end{tabular}

Sensitivity

Specificity = negativity in non-disease (61 patients $M-$ ). $=$ positivity in disease $(10$ patients $M+)$.

$\begin{array}{ll}\text { PV+ } & =\text { percent of positive test results due to disease. } \\ \text { PV - } & \text { = percent of negative test results due to non- }\end{array}$ disease. 
ease, (one patient) and cytotoxic therapy (one patient) at the time of initial examination.

If patients with metastases present at initial examination are considered as disease positive $(\mathrm{M}+)$ and those who showed no metastases throughout follow up disease negative $(\mathrm{M}-)$, the diagnostic value of the enzyme procedures can be calculated (Table).$^{10}$

There was no significant difference between $\gamma$-glutamyltransferase and biliary alkaline phosphatase activities by $\chi^{2}$ testing for any of the values shown.

\section{Discussion}

Our studies show that both the mini-column and electrophoretic procedures can be used to measure biliary alkaline phosphatase activity in plasma.

As shown by the examination of eluates, the mini-column ion exchange chromatographic method clearly separates the high molecular mass biliary from other alkaline phosphatase isoenzymes. The method is simple to perform and is reproducible, with a between batch coefficient of variation of about $10 \%$. It is rapid (working time for analysis of batch of 20 samples is less than $2 \mathrm{~h}$ ) and is inexpensive (reagent cost for a batch of 20 samples is about $£ 1 \cdot 50)$.

The electrophoretic method is also rapid (working time for analysis of batch of eight samples is 45 $\mathrm{min}$ ), and the reagent cost per batch of eight samples is about $£ 1 \cdot 50$. Reproducibility is also satisfactory (between batch coefficient of variation about $6 \%$ ). The electrophoretic method has the advantage that it permits the identification of altered activity of other alkaline phosphatase isoenzymes (for example, from bone) and avoids the possibility of false positives with the chromatographic method from the elution with biliary alkaline phosphatase of variant alkaline phosphatase isoenzymes, ${ }^{6}$ though these are rare.

Although the electrophoretic and chromatographic methods show excellent correlation, the electrophoretic method is twice as sensitive to biliary alkaline phosphatase. This must be a consequence of overestimation of biliary alkaline phosphatase activity by electrophoresis, since the chromatographic method yields about $82 \%$ recovery of total alkaline phosphatase and this recovery is independent of the percentage of the biliary fraction in the sample. It seems likely, as suggested by Crofton and Smith, ${ }^{6}$ that during staining of the electrophoresis membrane the high molecular mass of biliary alkaline phosphatase, compared with the other alkaline phosphatase isoenzymes, preferentially retards its diffusion into the substrate gel, so that staining of the cellulose acetate membrane at the site of biliary alkaline phosphatase is correspondingly enhanced. This suggestion was supported by the finding of a diminished proportion of biliary alkaline phosphatase staining in the substrate gel in comparison with that on the cellulose acetate membrane.

Biliary alkaline phosphatase was always shown chromatographically in samples showing biliary alkaline phosphatase by electrophoresis. This isoenzyme has never been detected by us by electrophoresis in sera of apparently healthy subjects. The detection limit for alkaline phosphatase by electrophoresis and visual inspection is 5 to $10 \mathrm{U} / \mathrm{l}$. All patients with biliary alkaline phosphatase visible by electrophoresis showed activity for this isoenzyme by scanning and by column of greater than $15 \mathrm{U} / \mathrm{l}$. Five of six samples with biliary alkaliné phosphatase undetectable by electrophoresis yielded values by mini-column of less than $20 \mathrm{U} / 1$, the higher figure presumably resulting from instrumental "noise" at the low enzyme activity values in the dilute column eluates. The reference limit for biliary alkaline phosphatase activity by the mini-column method may therefore be assumed to be about $20 \mathrm{U} / 1$ at $30^{\circ} \mathrm{C}$, and values above this figure can be regarded as increased.

Using the electrophoretic method we investigated the reported claim that biliary alkaline phosphatase is a more sensitive indicator than $\gamma$-glutamyltransferase for the detection of liver metastases. $^{8}$

Viot $e t a^{8}$ found that $97 \%$ of 75 patients with cancer with liver metastases had detectable biliary alkaline phosphatase but only $75 \%$ had a raised plasma $\gamma$-glutamyltransferase activity. In our patients biliary alkaline phosphatase and $\gamma$-glutamyltransferase had equal sensitivity and only $19 / 24(79 \%)$ of patients with liver metastases at the start of the study or during follow up showed increased biliary alkaline phosphatase or $\gamma$-glutamyltransferase activity at the time of diagnosis of metastases. Crofton $\mathrm{et} \mathrm{al}^{4}$ also found equal sensitivity of biliary alkaline phosphatase and $\gamma$-glutamyltransferase but found increased activity in all their 21 patients with liver metastases, whereas we report $21 \%$ false negatives.

It might be anticipated that biliary alkaline phosphatase would be more specific than $\gamma$-glutamyltransferase measurement because of lower sensitivity to alcohol and drug effects. Though a marginally superior specificity was found $(80 \%$ compared with 67\%), the difference was not significant. Six of the 12 patients who had no evidence of hepatic metastases but detectable biliary alkaline phosphatase activity in their plasma did 
have a possible alternative explanation for the presence of biliary alkaline phosphatase. In the rest, it is possible that high molecular mass alkaline phosphatase of biliary type might have originated from membrane fragments of the primary tumour containing alkaline phosphatase or from non-hepatic metastatic deposits. Post-mortem examination of two of these patients who died during the study showed no evidence of liver metastases.

In conclusion, we have described a simple and rapid ion exchange chromatographic method for the quantitative measurement of biliary alkaline phosphatase activity and have used it to evaluate an electrophoretic method. We used the latter to assess the value of biliary alkaline phosphatase measurement in patients with cancer investigated for possible hepatic metastases. For the detection of liver metastases both biliary alkaline phosphatase and $\gamma$-glutamyltransferase had similar sensitivity. Both were abnormal in about $80 \%$ of patients with liver metastases but abnormalities of biliary alkaline phosphatase were also found in $20 \%$ of patients without metasiases, and $33 \%$ of such patients had raised $\gamma$-glutamyltransferase activities. In less than half the patients with an abnormal value for either enzyme was this due to liver metastases. For the identification of liver metastases, therefore, the determinations have useful sensitivity but limited specificity.
References

' Price CP, Sammons HG. The nature of serum alkaline phosphatase in liver diseases. $J$ Clin Pathol 1974;27:392-8.

${ }^{2}$ De Broe ME, Borges M, Wieme RJ. The separation and characterisation of liver plasma membrane fragments circulating in the blood of patients with cholestasis. Clin Chim Acta 1975;59:369-72.

${ }^{3}$ Viot M, Joulin C, Cambon P, Krebs BP, Schneider M, Lelanne $\mathrm{CM}$. The value of serum alkaline phosphatase $\alpha$ isoenzyme in the diagnosis of liver metastases. Biomedicine 1979;31:74-7.

${ }^{4}$ Crofton PM, Elton RA, Smith AF. High molecular weight alkaline phosphatase: A clinical study. Clin Chim Acto 1979;98:263-75.

${ }^{5}$ Rhone DP, Mizuno FM. Profiles of alkaline phosphatase isoenzymes in serum using cellulose acetate electrophoresis and organ specific inhibitors. Am J Clin Pathol 1973;59:531-41.

- Crofton PM, Smith AF. An ion exchange assay for high molecular weight alkaline phosphatase. Clin Chim Acta 1979;98:253-61.

' Anido G, Soto A, McBeth CH, Romero P. Alkaline phosphatase isoenzyme in diagnosis. Quad Sclavo Diagn 1972;8:541-52.

${ }^{8}$ Viot M, Thyss A, Viot G, et al.Comparative study of gamma glutamyltransferase, alkaline phosphatase and its $\alpha_{1}$ isoenzyme as biological indicators of liver metastases. Clin Chim Acta 1981;115:349-58.

' Rosalki SB. Co-ordinator. Association of Clinical Biochemists' Working Party recommendations: Proposed methods for determinations of some enzymes in blood serum. Association of Clinical Biochemists News Sheet (Suppl). February 1980: 9-12.

${ }^{10}$ Vecchio RJ. Predictive value of a single diagnostic test in unselected population. $N$ Engl J Med 1966;274:1171-3.

Requests for reprints to: Dr S B Rosalki, Consultant Chemical Pathologist, Department of Chemical Pathology, The Royal Free Hospital, Pond Street, Hampstead, London NW3 2QG, England. 